\title{
Treating the Lips and Its Anatomical Correlate in Respect to Vascular Compromise
}

\author{
Sahar Ghannam, MD, PhD ${ }^{1,2}$ Sonja Sattler, $\mathrm{MD}^{3} \quad$ Konstantin Frank, MD ${ }^{4}$ David L. Freytag ${ }^{4}$ \\ Katherine L. Webb ${ }^{5}$ Aditya Devineni ${ }^{5}$ Sebastian Cotofana, MD, PhD ${ }^{5}$
}

${ }^{1}$ Director Sahar Polyclinic, Kuwait

2 Department of Dermatology, Venereology and Andrology, University of Alexandria, Egypt

3 Rosenpark Klinik, Darmstadt, Germany

4 Department for Hand, Plastic and Aesthetic Surgery,

Ludwig - Maximilian University Munich, Germany

5 Department of Medical Education, Albany Medical College, Albany,

New York

Facial Plast Surg 2019;35:193-203.
Address for correspondence Sebastian Cotofana, MD, PhD, Associate Professor, Albany Medical College, 47 New Scotland Avenue MC-135, Albany, NY 12208 (e-mail: cotofas@amc.edu).

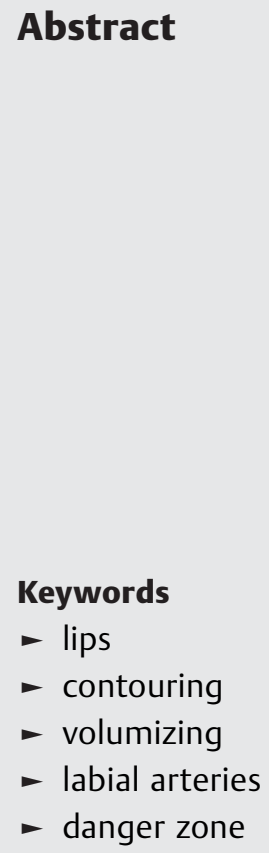

Treating the lips to increase facial attractiveness and youthfulness is challenging when trying to consider ethnic differences in an increasingly more diverse society. Multiple injection techniques are currently available for treating lip contour and volume, but a validation in the cadaveric model under the aspects of safety has not been performed yet. The injection techniques presented in this study are based on the experience and personal selection of the authors. The authors have assessed, treated, and evaluated for more than 20 years patients from the Middle East and Central Europe. Cadaveric verification was performed for each of the presented techniques to identify the positioning of the injected product inside the lips and its relation to the superior/inferior labial arteries. The results of the anatomic analyses revealed that in $58.3 \%$ of the performed injections, the product was placed close to the superior/inferior labial arteries. In $60.0 \%$ of the cases, applications using a needle placed the injected product in endangered locations, whereas $57.1 \%$ of the cases using cannulas placed the product in endangered locations (i.e., in the vicinity of the superior/inferior labial arteries). This anatomic study revealed that injected material into the lips is frequently placed in close proximity to labial arteries representing a high risk for intra-arterial applications, leading to tissue loss (necrosis) and potential end-arterial embolism (potential blindness). Nevertheless, treatment of the lips should be a multistep approach focusing first on the far (upper and middle face) and close (labiomandibular and labiomental) perioral regions.
Lips are the central point of focus in the lower face and have always played a key role in facial attractiveness. Besides their function as a perioral sphincter (together with the perioral muscular complex), lips are majorly involved in the expression of emotions, speech building, and food intake. The human lips have evolved during various evolutionary steps when compared with the lips of apes: they have increased in volume and the vermillion became more everted. ${ }^{1}$ The eversion of the lips is thought to increase the contrast between the white/brown perioral skin, and the increased volume is thought to

Issue Theme Aesthetic Treatment of the Jawline and Perioral Area; Guest Editor: S. Randolph Waldman, MD facilitate nonverbal communication and thus increase attractiveness. $^{1}$

In today's society, these features, contrasting pigment color and lip fullness, are still considered to increase facial attractiveness and have become a major area of interest. Using lipstick increases the contrast between the lips and the perioral skin, whereas using soft-tissue fillers increases the lip volume. The demand for the latter (soft-tissue filler volumization) is increasing independent of gender, ethnic background, or age. Hyaluronic acid-based soft-tissue fillers are one of the most used agents for this type of treatment. ${ }^{2-11}$
Copyright $\odot 2019$ by Thieme Medical Publishers, Inc., 333 Seventh Avenue, New York, NY 10001, USA. Tel: +1(212) 584-4662.
DOI https://doi.org/ 10.1055/s-0039-1683856. ISSN 0736-6825. 
On the contrary, loss of lip contour, lip volume, and/or presence of perioral wrinkles are considered signs of facial aging and have been associated with reduced attractiveness. ${ }^{12,13}$ Reducing the signs of lip aging is crucial to restore facial attractiveness. Multiple techniques to restore facial aging via the treatment of the lips have been described. ${ }^{14-19}$ However, few of these techniques have been validated for the positioning of the injected product in relation to the superior/inferior labial arteries. It is widely accepted that injection of soft-tissue filler material into the arterial system is associated with tissue loss (necrosis) and potential endarterial embolism (potential blindness). ${ }^{20-23}$

The present study describes and summarizes injection techniques targeting the lips with hyaluronic acid-based soft-tissue fillers, which are frequently used in Middle Eastern and Caucasian patients. The injection techniques included in this anatomical study are based on the experience of the authors, each with more than 20 years of experience treating signs of facial aging and the lips using soft-tissue fillers. The included injection techniques were verified in the cadaveric model for their positioning within the upper/lower lip in relation to the superior/inferior labial arteries.

\section{Material and Methods}

\section{Injection Techniques}

The injection techniques presented in this study are based on the experience and personal selection of the authors. The authors have assessed, treated, and evaluated for more than 20 years patients from the Middle East and Central Europe. Based on individual algorithms of the authors, some of the most frequently used injection techniques are presented in the following.

The injection techniques presented include the usage of a $27 \mathrm{G} \times 1 / 2$ " and $30 \mathrm{G} \times 1$ " sharp needle (various companies) and $22 \mathrm{G} \times 2$ " and $25 \mathrm{G} \times 1$ 1/2" blunt tip cannula (various companies). Various injection points were used and each technique was tailored in application and amount of injected product based on patients' needs and clinical indication.

\section{Anatomical Verification}

The same injection techniques as used by the authors in the (living) patients were performed in the upper and in the lower lips of three fresh cadaveric specimens ( $1 \mathrm{x}$ male / $2 \mathrm{x}$ females) having a mean age of $84.67 \pm 10.02$ years and a body mass index of $26.18 \pm 5.45 \mathrm{~kg} / \mathrm{m}^{2}$. Specimens were screened and not included into this analysis if previous facial surgery or diseases disrupted the integrity of the facial anatomy. Each body donor had given informed consent while alive for the use of his or her body for medical, scientific, and educational purposes. All aspects of the study conform to the laws of the country where the study was conducted.

The injected product (for the cadaveric injections) was commercially available hair gel mixed with commercially available food coloring to visualize the injected product inside the cadaveric tissue. The superior and inferior labial arteries were previously injected with red latex, to visualize the arterial vascular system of the lips. Cross-sectional dissections and photo-imaging was performed to identify the placement of the injected colored product inside the lips and to relate the position of the product to the superior/inferior labial arteries.

\section{Results}

\section{Contouring}

Technique: Lip Contouring I (- Fig. 1)

Injector: $27 \mathrm{G}$ needle

Injection point: Highest point of cupid's arch

Positioning of material: Subdermal in retrograde 2 to 3

single lines; medial to lateral

Amount of product: $0.05 \mathrm{cc}$ per line

Anatomical analysis: The product was positioned in the subcutaneous plane of the vermillion. The artery was located in the submucosal position and was separated from the product by the orbicularis oris muscle.

\section{Technique: Lip Contouring II (-Fig. 2)}

Injector: $30 \mathrm{G}$ needle

Injection point: Skin $5 \mathrm{~mm}$ lateral to the corner of the mouth at the modiolus

Positioning of material: Subdermal in retrograde 2 to 3 single lines for the upper lip and 1 line for the lower lip (lower lip before upper lip); lateral to medial

Amount of product: $0.05 \mathrm{cc}$ per line

Anatomical analysis: The product was positioned in the subcutaneous plane of the vermillion. The artery was located in the submucosal position and was separated from the product by the orbicularis oris muscle.

\section{Volumizing Upper Lip}

\section{Technique: Lip Volumizing Upper Lip I (- Fig. 3)}

Injector: $30 \mathrm{G}$ needle Injection point: Vermillion border of upper lip Positioning of material: Submucosal bolus deep inside the upper lip with multiple entry points (4-6) in oblique direction

Amount of product: $0.05 \mathrm{cc}$ per bolus

Anatomical analysis: The product was positioned in the submucosal plane in close relationship to the artery. In the present dissection, two submucosal arteries were present increasing the risk for an intravascular application.

\section{Technique: Lip Volumizing Upper Lip II (-Fig. 4)}

Injector: $27 \mathrm{G}$ needle

Injection point: Skin of upper lip 3 to $7 \mathrm{~mm}$ superior to vermillion border

Positioning of material: Submucosal bolus deep inside the upper lip with multiple entry points (4-6) in straight direction

Amount of product: $0.05 \mathrm{cc}$ per bolus

natomical analysis: The product was positioned in the submucosal plane in close relationship to the artery. In the present dissection two arteries were identified: 

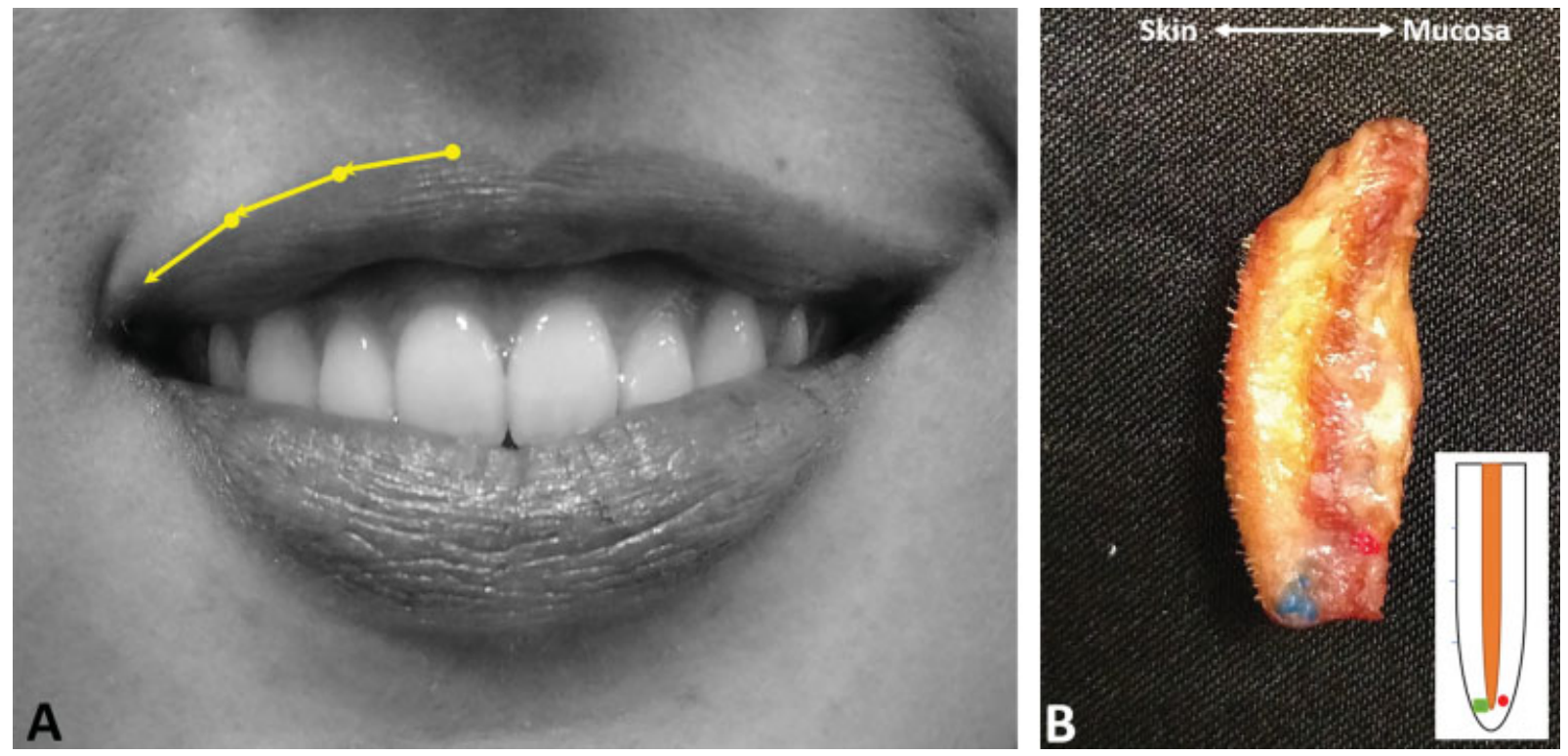

Fig. 1 (A, B) Part A shows the lips of a patient. The colored arrows indicate the point of injection and the direction of the product displacement. The yellow color represents the treatment with a needle. Part B shows a cross-section of an injected lip. The blue color represents the stained injected product. The red color represents the arteries. The small box in the right lower corner shows a schematic drawing of the lip, the product and the artery, and their position toward each other. Note: In this case, the artery is located in the submucosal plane and the product is located in the subcutaneous plane. There is no close relationship between the product and the artery.
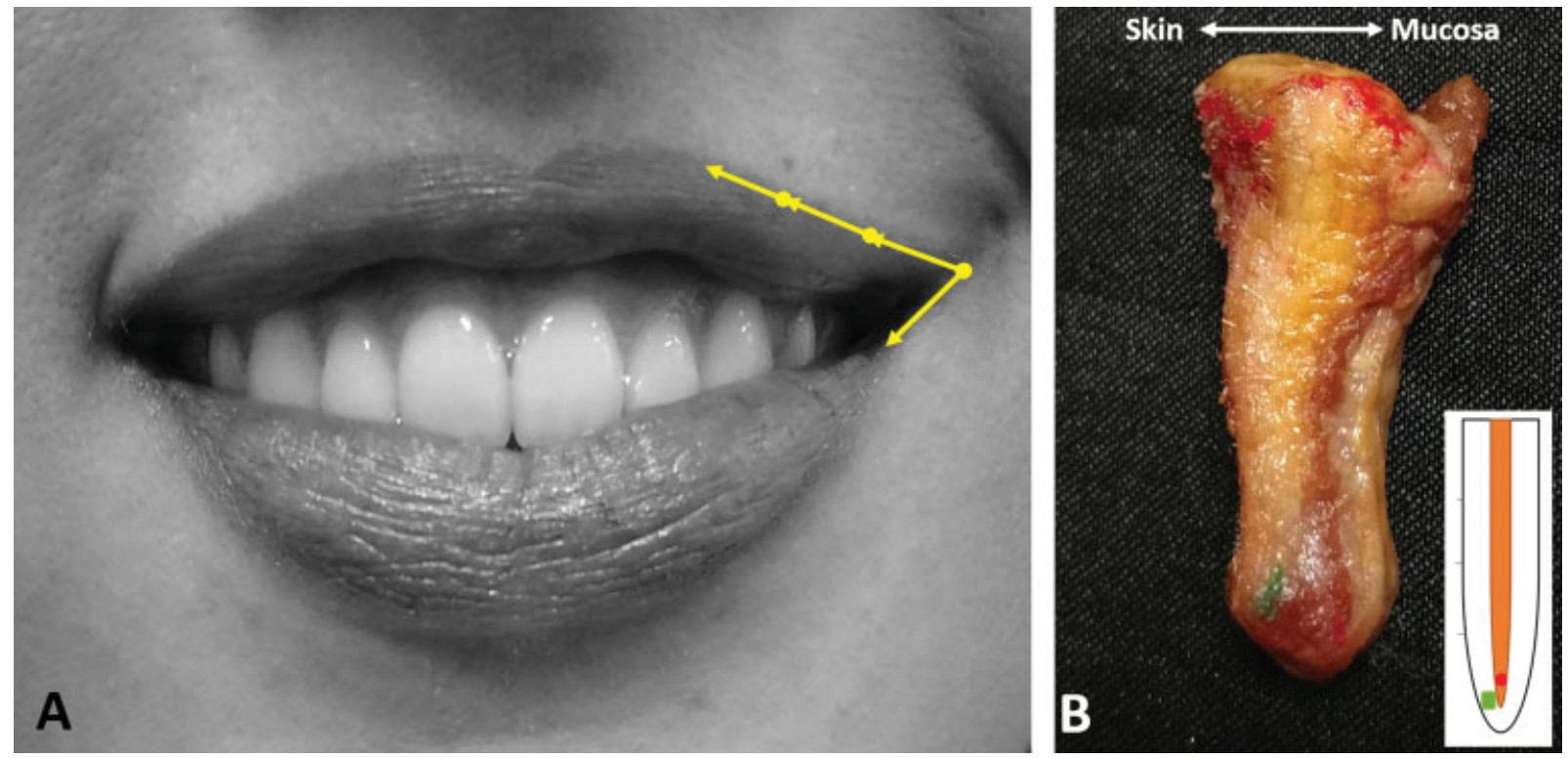

Fig. 2 (A, B) Part A shows the lips of a patient. The colored arrows indicate the point of injection and the direction of the product displacement. The yellow color represents the treatment with a needle. Part B shows a cross-section of an injected lip. The green color represents the stained injected product. The red color represents the arteries. The small box in the right lower corner shows a schematic drawing of the lip, the product and the artery, and their position toward each other. Note: In this case, the artery is located in the intramuscular plane and the product is located in the subcutaneous plane. There is no close relationship between the product and the artery.

subcutaneous and intramuscular position. This rare positioning of the arteries is increasing the risk for an intravascular application.

\section{Technique: Lip Volumizing Upper Lip III (-Fig. 5)}

Injector: $25 \mathrm{G}$ cannula

Injection point: Vermillion border at the corner of the mouth (skin-vermillion-transition)
Positioning of material: Submucosal application of product in a retrograde line below vermillion border starting at the cupid's arch

Amount of product: $0.20 \mathrm{cc}$ per line

Anatomical analysis: The product was positioned in the submucosal plane of the vermillion in a wide range between the vermillion border and the beginning of the oral mucosa. In the present case, multiple arteries 

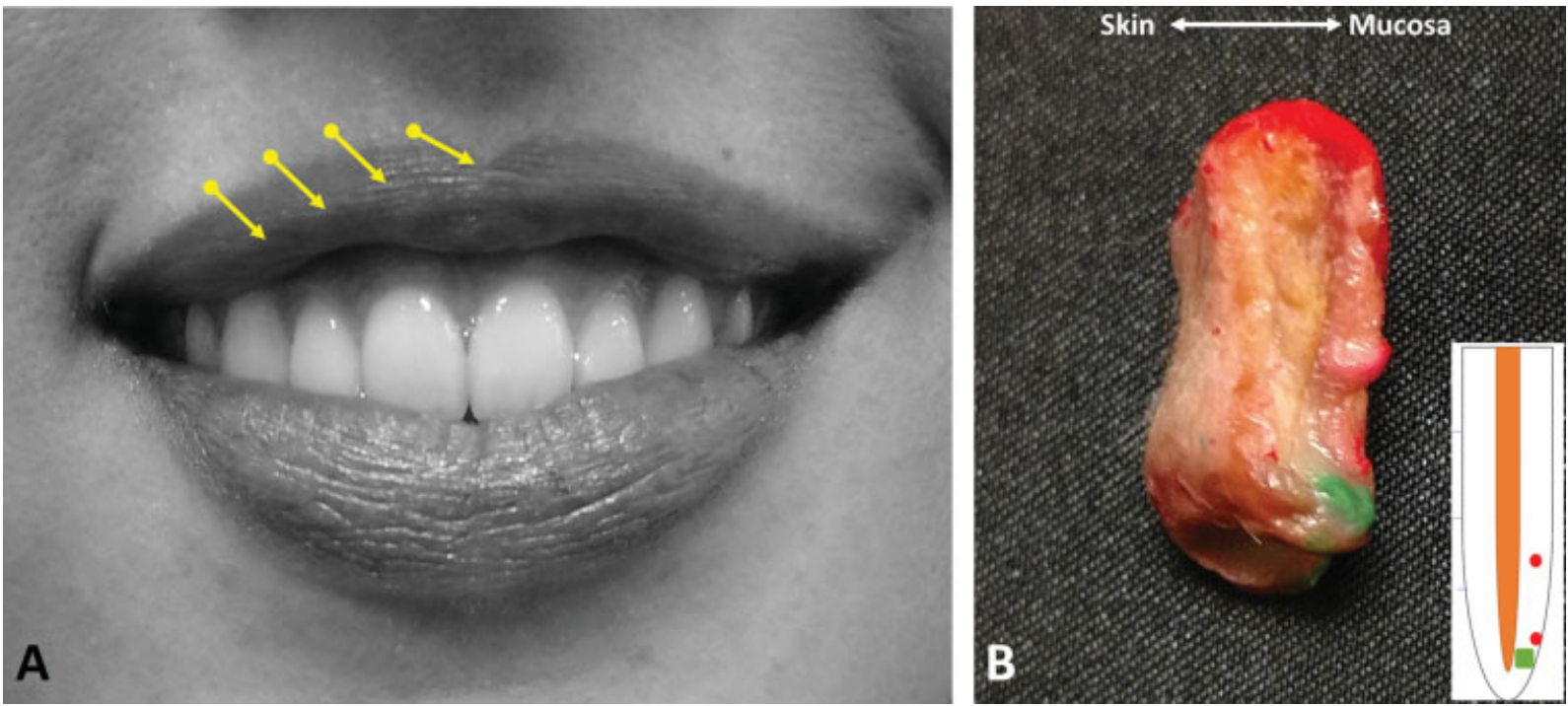

Fig. 3 (A, B) Part A shows the lips of a patient. The colored arrows indicate the point of injection and the direction of the product displacement. The yellow color represents the treatment with a needle. Part B shows a cross-section of an injected lip. The green color represents the stained injected product. The red color represents the arteries. The small box in the right lower corner shows a schematic drawing of the lip, the product and the artery, and their position toward each other. Note: In this case, there are two arteries. Both are located in the submucosal plane and the product is also located in the submucosal plane. The product and the arteries are in close relation to each other.
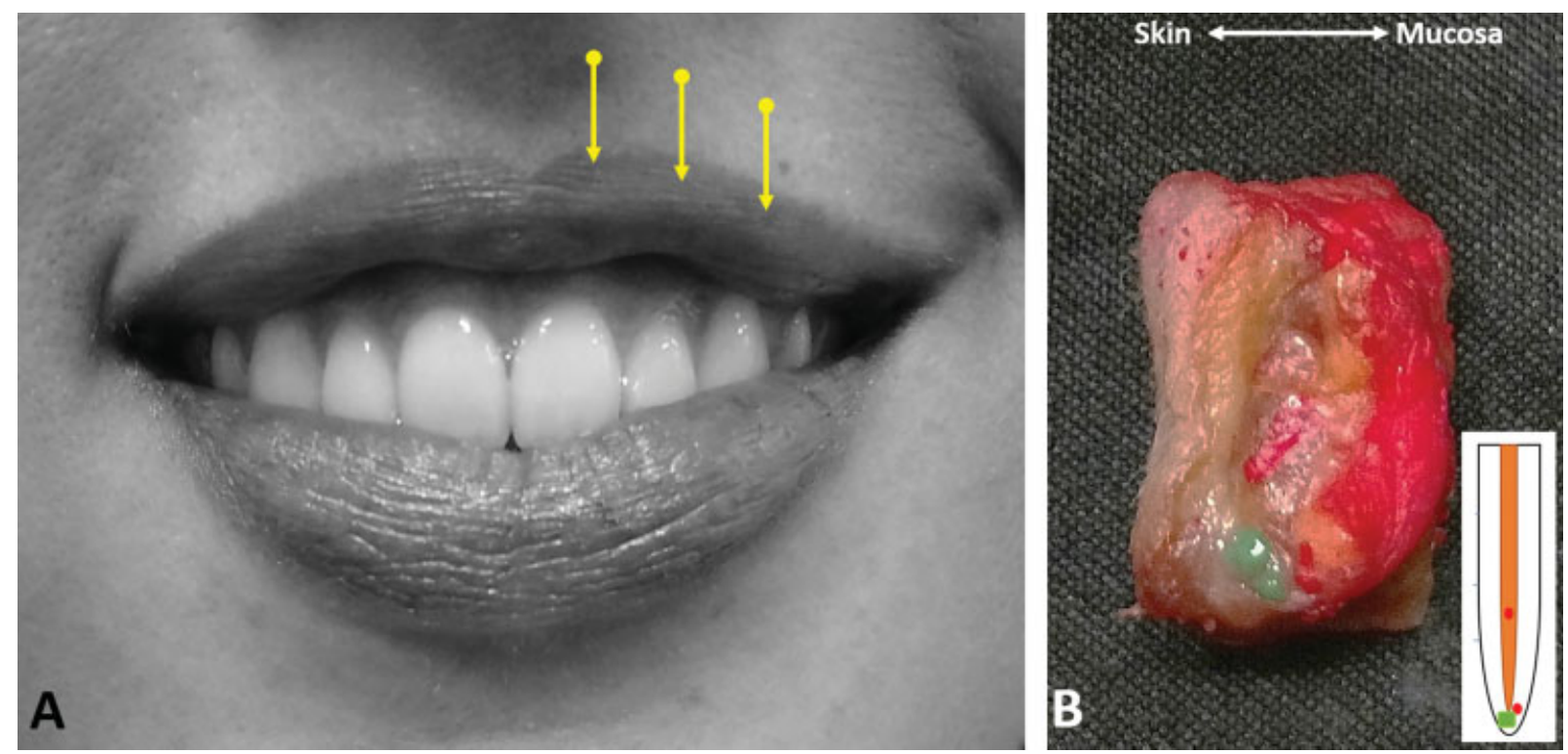

Fig. 4 (A, B) Part A shows the lips of a patient. The colored arrows indicate the point of injection and the direction of the product displacement. The yellow color represents the treatment with a needle. Part B shows a cross-section of an injected lip. The green color represents the stained injected product. The red color represents the arteries. The small box in the right lower corner shows a schematic drawing of the lip, the product and the artery, and their position toward each other. Note: In this case, there are two arteries. One is located in the intramuscular plane. The other one is located in the submucosal plane. The product is located in the vermillion. The product and the arteries are in close relation to each other.

were present both in the submucosal and in subcutaneous position. However, the product was applied superficially, thus being in distinct distance to the arteries.

\section{Technique: Lip Volumizing Upper Lip IV (-Fig. 6)}

Injector: $25 \mathrm{G}$ cannula

Injection point: Vermillion border at the corner of the mouth (skin-vermillion-transition)
Positioning of material: Submucosal application of product in a retrograde line in the vermillion border (white roll) starting at the cupid's arch

Amount of product: $0.20 \mathrm{cc}$ per line

Anatomical analysis: The product was positioned in the submucosal plane of the vermillion in a wide range between the vermillion border and the beginning of the oral mucosa. The artery was present in the subcutaneous position in relatively close relationship to the product. 

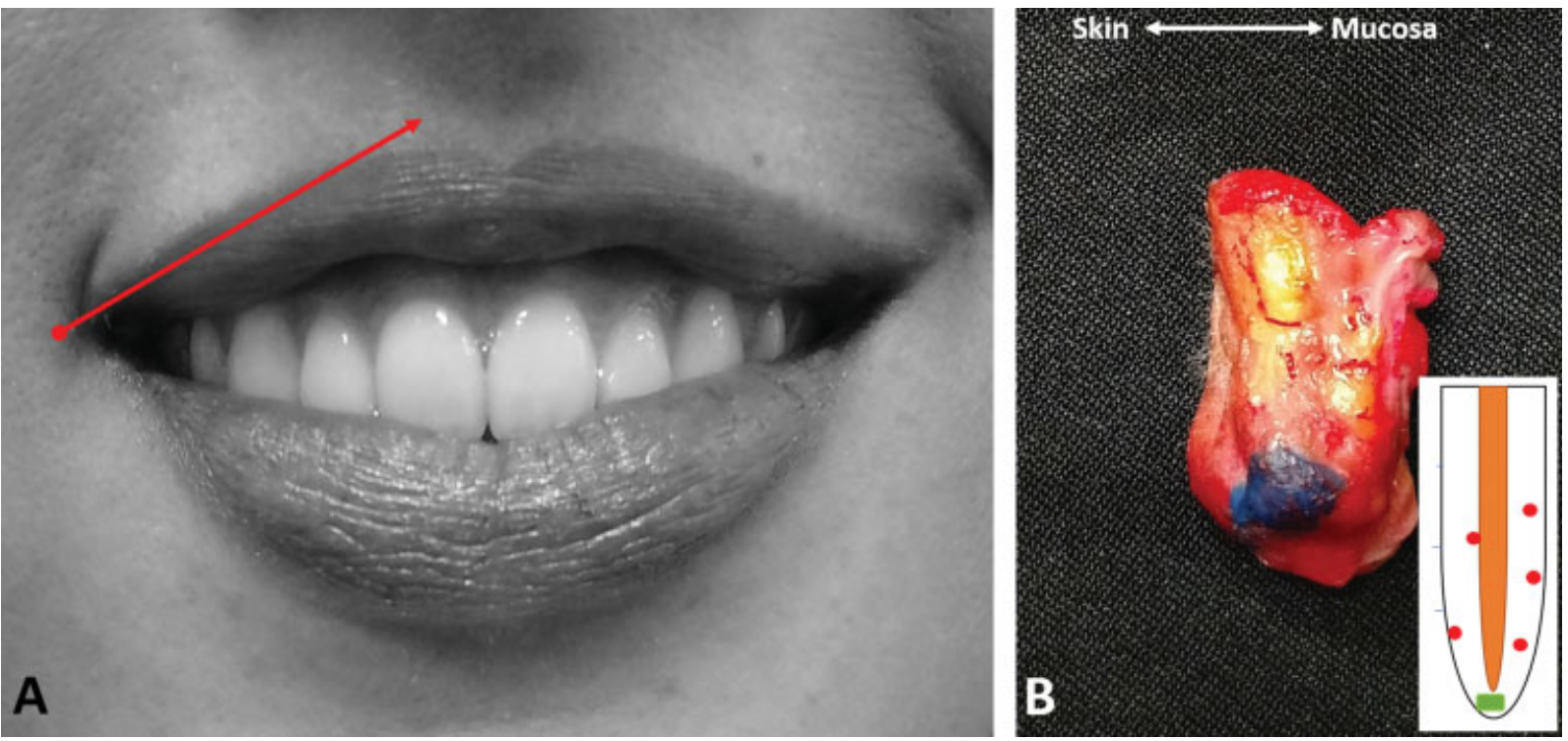

Fig. 5 (A, B) Part A shows the lips of a patient. The colored arrow indicates the point of injection and the direction of the product displacement. The red color represents the treatment with a cannula. Part B shows a cross-section of an injected lip. The blue color represents the stained injected product. The red color represents the arteries. The small box in the right lower corner shows a schematic drawing of the lip, the product and the artery, and their position toward each other. Note: In this case, there are several arteries, found in the submucosal and the subcutaneous plane. The product is located in the vermillion. The product and the arteries are in no close relation to each other.

\section{Volumizing Lower Lip}

\section{Technique: Lip Volumizing Lower Lip I (-Fig. 7)}

Injector: 30G needle

Injection point: Vermillion border of lower lip

Positioning of material: Submucosal bolus deep inside the lower lip with multiple entry points (1-3) in straight direction

Amount of product: $0.05 \mathrm{cc}$ per bolus
Anatomical analysis: The product was positioned in the submucosal plane in close relationship to the artery increasing the risk for an intravascular application.

\section{Technique: Lip Volumizing Lower Lip II (-Fig. 8)}

Injector: 27G needle

Injection point: Skin of lower lip 3 to $7 \mathrm{~mm}$ inferior to vermillion border

Positioning of material: Submucosal bolus deep inside the lower lip with multiple entry points (1-3) in straight direction
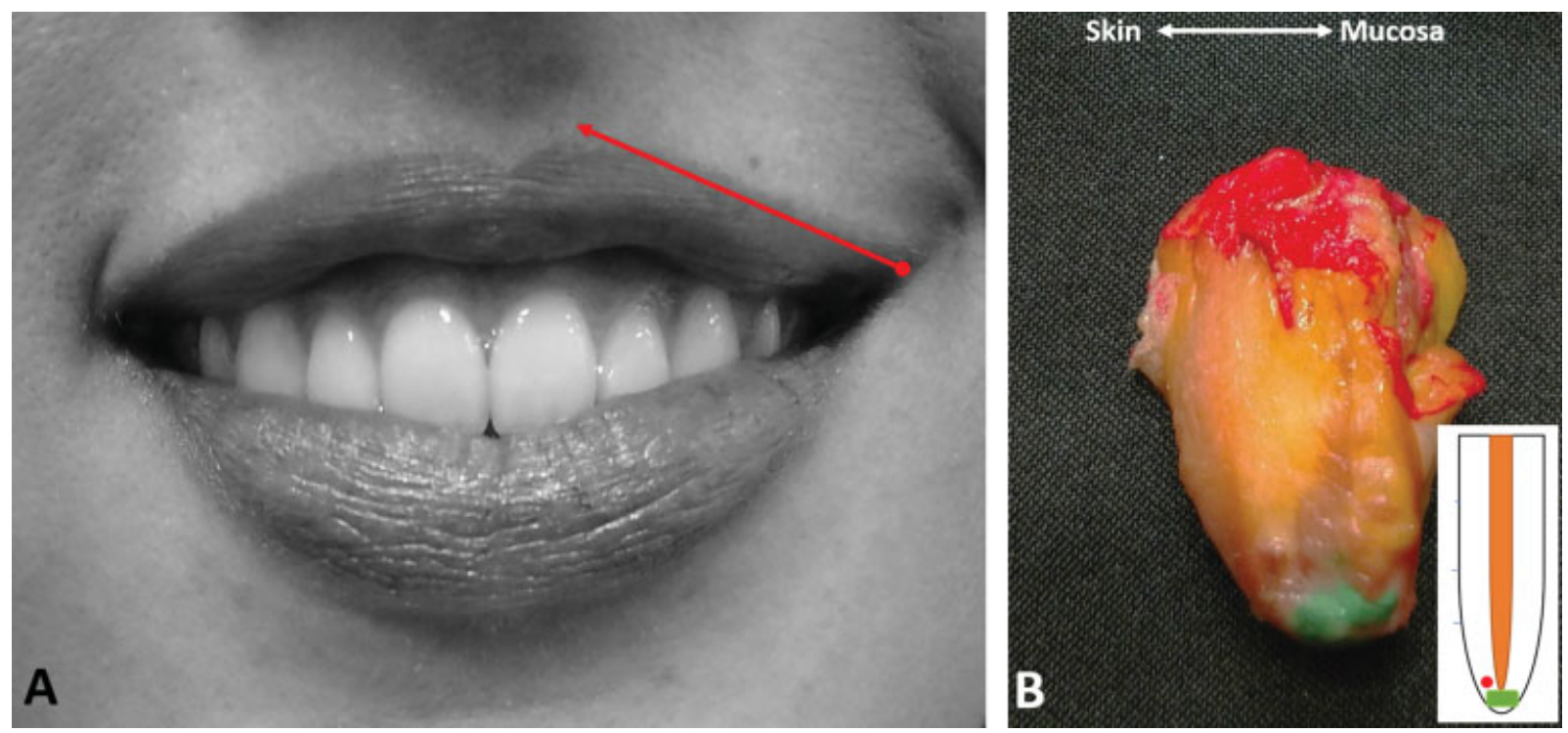

Fig. 6 (A, B) Part A shows the lips of a patient. The colored arrow indicates the point of injection and the direction of the product displacement. The red color represents the treatment with a cannula. Part B shows a cross-section of an injected lip. The green color represents the stained injected product. The red color represents the arteries. The small box in the right lower corner shows a schematic drawing of the lip, the product and the artery, and their position toward each other. Note: In this case, the artery is located in the subcutaneous plane. The product is located in the vermillion. The product and the artery are in close relation to each other. 

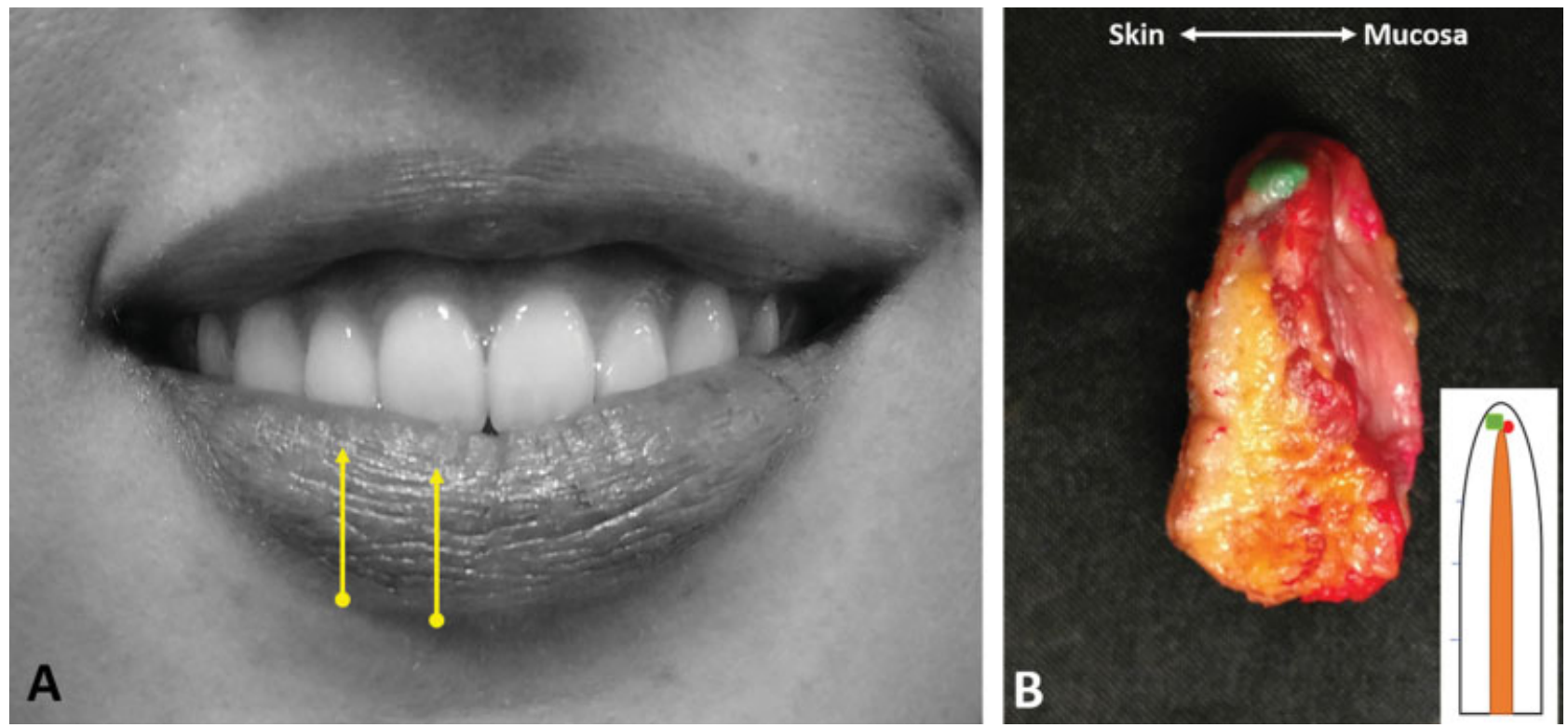

Fig. 7 (A, B) Part A shows the lips of a patient. The colored arrows indicate the point of injection and the direction of the product displacement. The yellow color represents the treatment with a needle. Part B shows a cross-section of an injected lip. The green color represents the stained injected product. The red color represents the arteries. The small box in the right lower corner shows a schematic drawing of the lip, the product and the artery, and their position toward each other. Note: In this case, the artery is located in the submucosal plane. The product is located in the vermillion. The product and the artery are in close relation to each other.

Amount of product: $0.05 \mathrm{cc}$ per bolus

Anatomical analysis: The product was positioned in the submucosal plane (deep to the orbicularis oris muscle) in close relationship to the artery increasing the risk for an intravascular application. In this case, multiple arteries were identified in the submucosal and subcutaneous plane.
Technique: Lip Volumizing Lower Lip III (-Fig. 9)

Injector: 25G cannula

Injection point: Vermillion border at the corner of the mouth (skin-vermillion-transition)

Positioning of material: Submucosal application of product in a retrograde line in the vermillion border (white
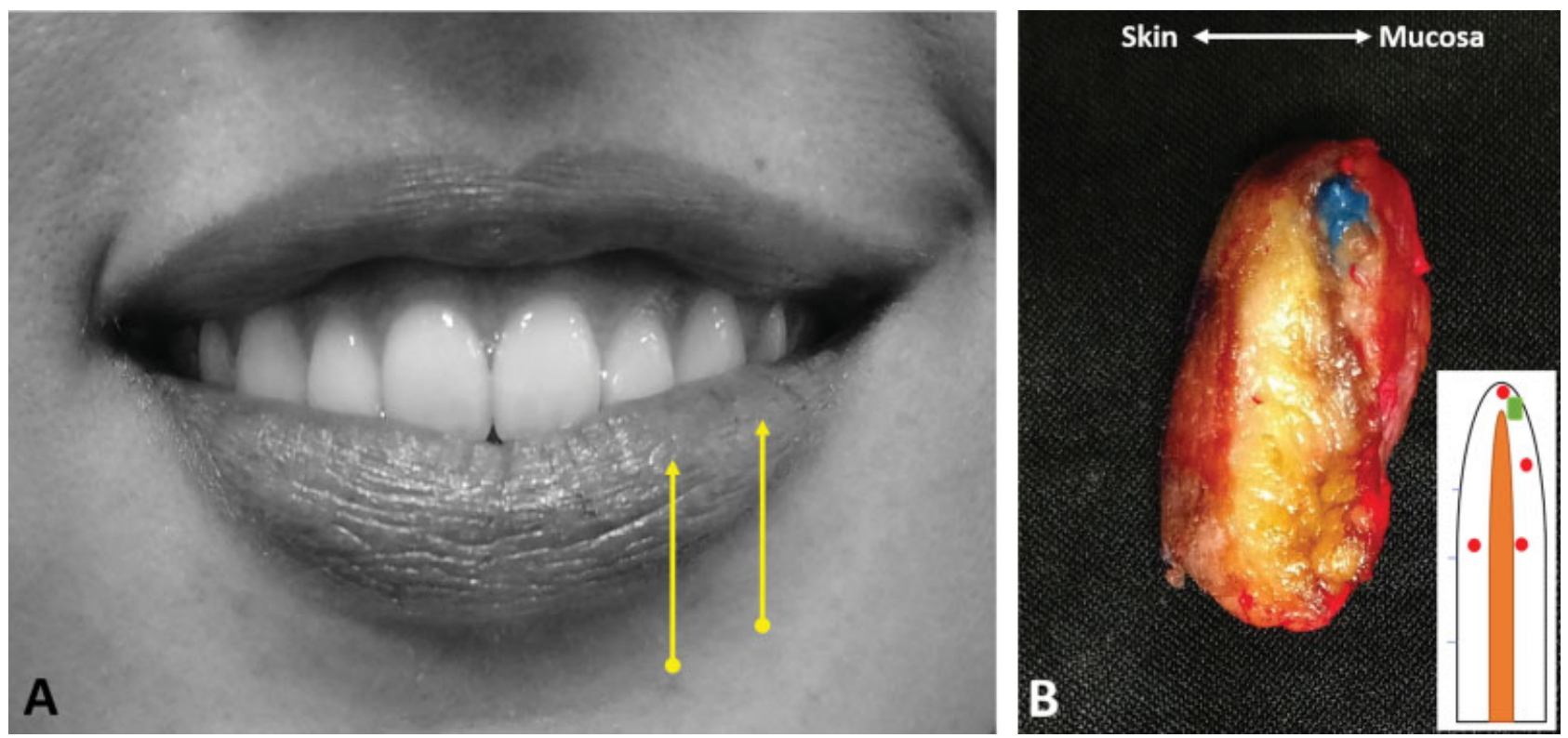

Fig. 8 (A, B) Part A shows the lips of a patient. The colored arrows indicate the point of injection and the direction of the product displacement. The yellow color represents the treatment with a needle. Part B shows a cross-section of an injected lip. The blue color represents the stained injected product. The red color represents the arteries. The small box in the right lower corner shows a schematic drawing of the lip, the product and the artery, and their position toward each other. Note: In this case, there are several arteries, located in the submucosal and the subcutaneous plane and in the vermillion. The product is located in the submucosal plane. The product and one artery are in close relation to each other. 

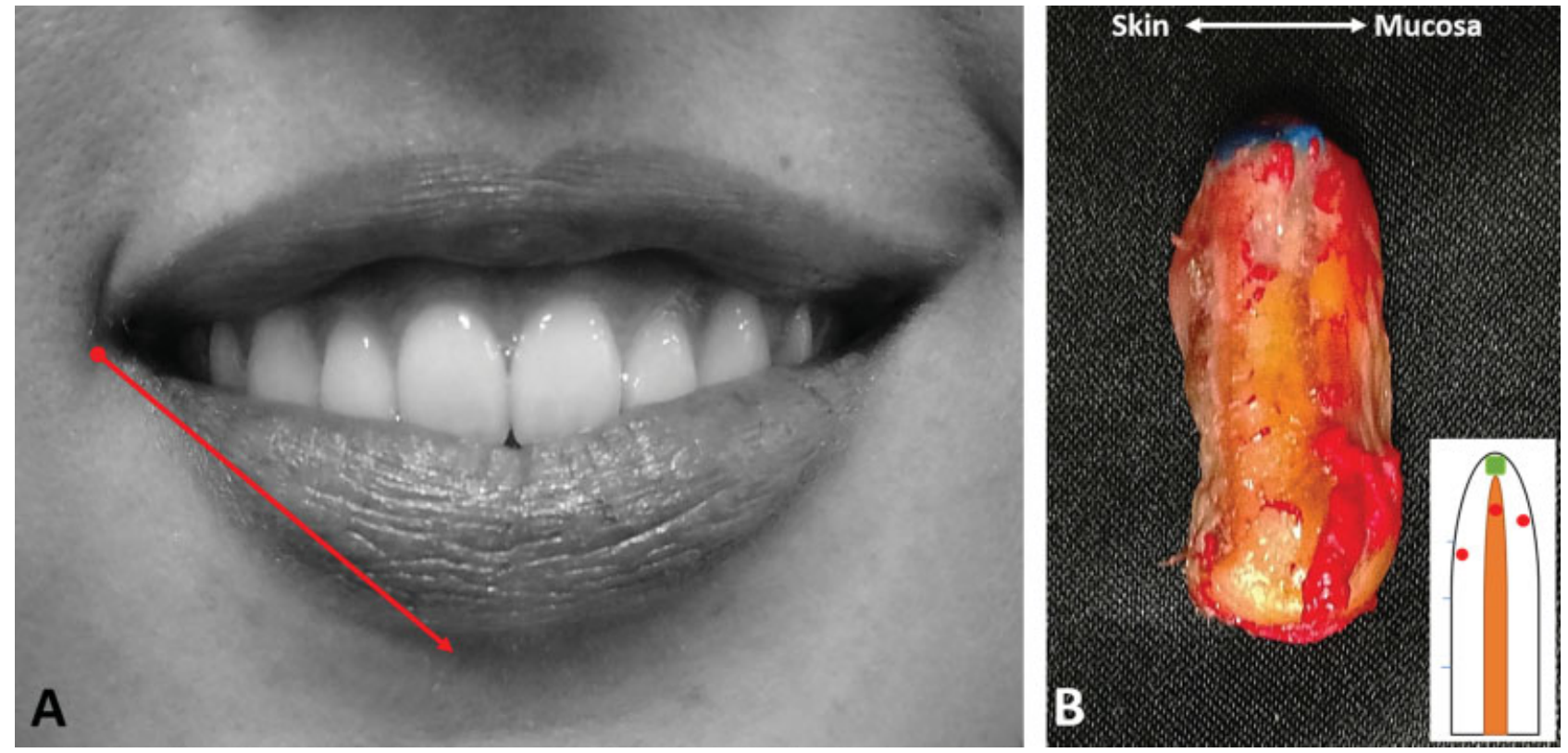

Fig. 9 (A, B) Part A shows the lips of a patient. The colored arrow indicates the point of injection and the direction of the product displacement. The red color represents the treatment with a cannula. Part B shows a cross-section of an injected lip. The blue color represents the stained injected product. The red color represents the arteries. The small box in the right lower corner shows a schematic drawing of the lip, the product and the artery, and their position toward each other. Note: In this case, there are several arteries, located in the submucosal, intramuscular and subcutaneous plane. The product is located in the vermillion. The product and the arteries are in close relation to each other.

roll) starting at the body of the lower (ipsilateral) lip (paramedian).

Amount of product: $0.15 \mathrm{cc}$ per line

Anatomical analysis: The product was positioned in the submucosal plane of the vermillion in a wide range between the vermillion border and the beginning of the oral mucosa. In the present case, multiple arteries were present in the submucosal, intramuscular, and the subcutaneous position. The product was positioned in close relationship to the arteries in this case.

\section{Technique: Lip Volumizing Lower Lip IV (-Fig. 10)}

Injector: 25G cannula

Injection point: Vermillion border at the corner of the mouth (skin-vermillion-transition)

Positioning of material: Submucosal application of product in a retrograde line deep inside the vermillion starting at the body of the lower lip of the contralateral side of the lip (crossing the midline). Close to the corner of the mouth, a small bolus of $0.01 \mathrm{cc}$ will be applied to provide support for the modiolus

Amount of product: $0.3 \mathrm{cc}$ per line

Anatomical analysis: The product was positioned deep inside the lower lip in close relationship to the oral mucosa. The arteries were located anterior to the product in the subcutaneous and intramuscular plane. The product was positioned in close relationship to the arteries in this case increasing the risk for intra-arterial application of the product.

\section{Perioral Lower Lip}

\section{Technique: Labiomental Volumization I (-Fig. 11)}

Injector: $27 \mathrm{G}$ needle

Injection point: Skin of lower lip 3 to $7 \mathrm{~mm}$ inferior to vermillion border

Positioning of material: Deep intramuscular bolus inside the lower lip with multiple entry points (1-3) in oblique direction

Amount of product: 0.02 to $0.05 \mathrm{cc}$ per bolus

Anatomical analysis: The product was positioned in the intramuscular plane (inside the orbicularis oris muscle). The artery was identified in a more cranial position (note the change in planes of the inferior labial artery in this case).

\section{Technique: Labiomental Volumization II (-Fig. 12)}

Injector: $22 \mathrm{G}$ cannula

Injection point: Skin of lower lip 10 to $30 \mathrm{~mm}$ inferolateral to vermillion border

Positioning of material: Deep intramuscular lines inside the lower lip in fanning technique

Amount of product: 0.02 to $0.05 \mathrm{cc}$ per line

Anatomical analysis: The product was positioned in the intramuscular plane (inside the orbicularis oris muscle). The arteries were identified in a more cranial position being located in the submucosal and in the intramuscular plane. 

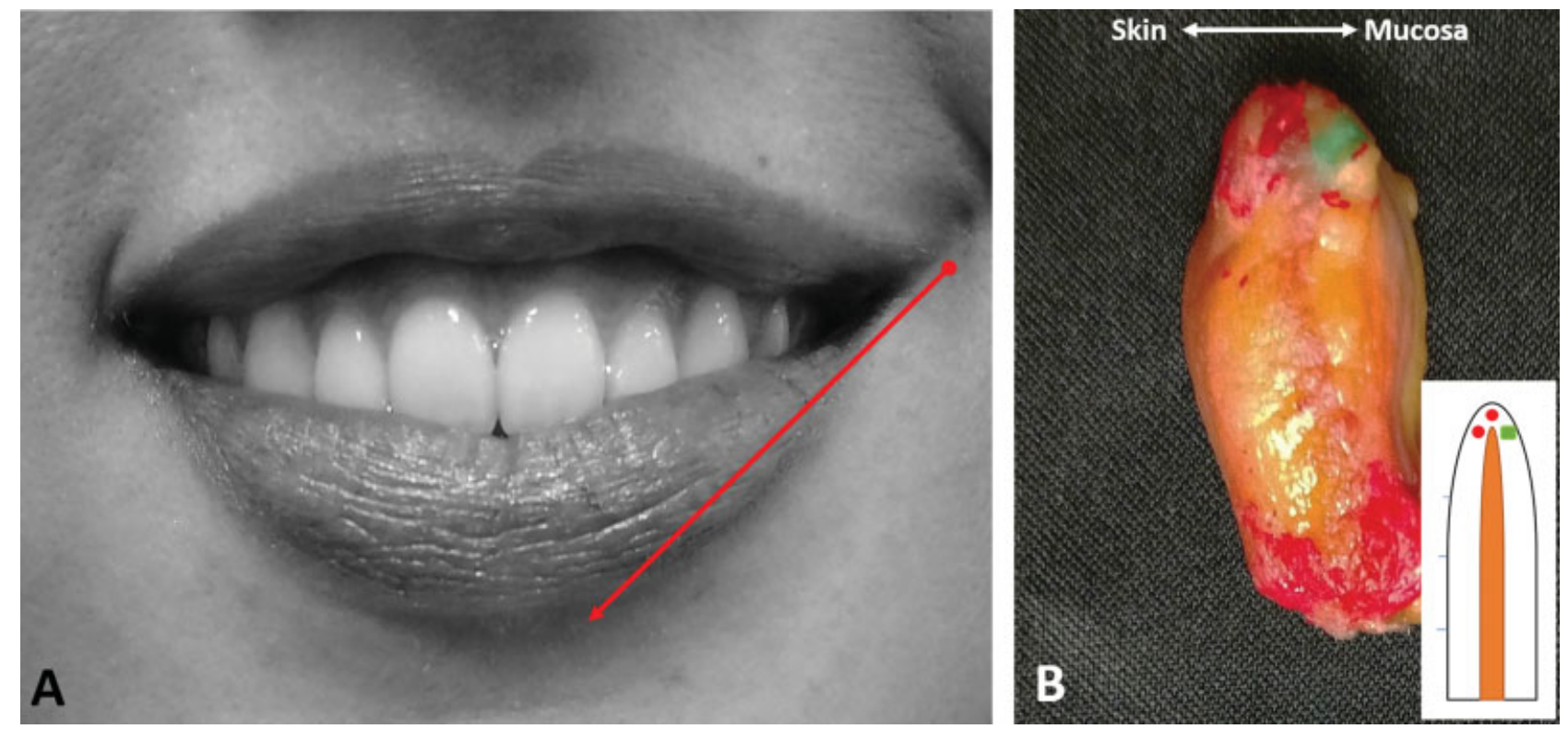

Fig. 10 (A, B) Part A shows the lips of a patient. The colored arrow indicates the point of injection and the direction of the product displacement. The red color represents the treatment with a cannula. Part B shows a cross-section of an injected lip. The green color represents the stained injected product. The red color represents the arteries. The small box in the right lower corner shows a schematic drawing of the lip, the product and the artery, and their position toward each other. Note: In this case, there are two arteries. One is located in the subcutaneous plane. The other one is located in the vermillion. The product is located in the submucosal plane. The product and the arteries are in close relation to each other.

\section{Discussion}

This study presented 12 different injection techniques frequently used in Middle Eastern and Central European patients when addressing the lips for aesthetic purposes. The presented techniques included contouring (using a needle), volumizing (using both needles and cannulas), and labiomental treatment (using both needles and cannulas) for labiomental volume loss. Cadaveric verification was performed for each of the presented techniques to identify the positioning of the injected product inside the superior/ inferior lip. The results of the anatomic analyses revealed that in $58.3 \%$ of the performed injections the product was placed in close proximity to the superior/inferior labial
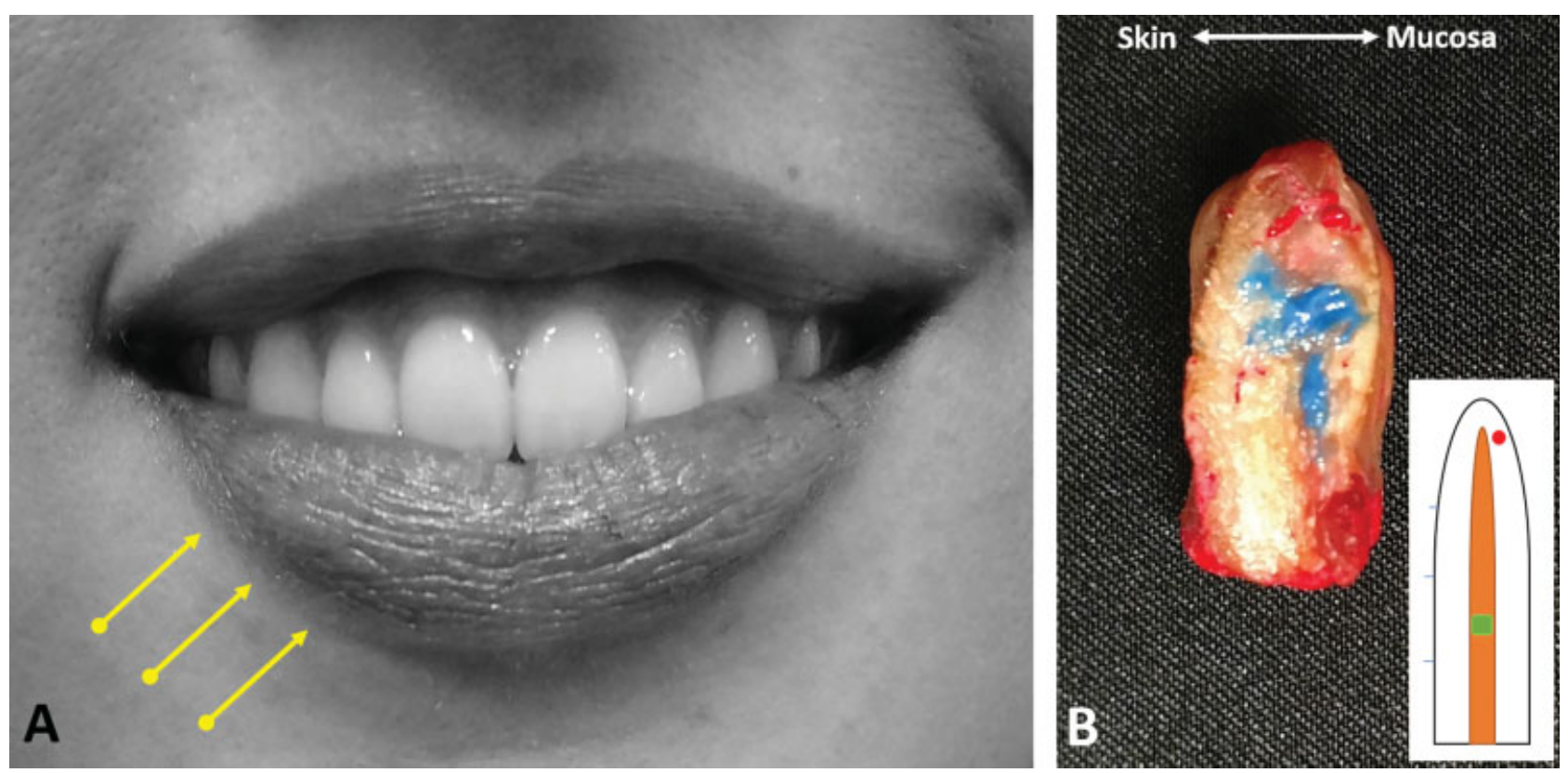

Fig. 11 (A, B) Part A shows the lips of a patient. The colored arrows indicate the point of injection and the direction of the product displacement. The yellow color represents the treatment with a needle. Part B shows a cross-section of an injected lip. The blue color represents the stained injected product. The red color represents the arteries. The small box in the right lower corner shows a schematic drawing of the lip, the product and the artery, and their position toward each other. Note: In this case, the artery is located in the submucosal plane and the product is located in the intramuscular plane. There is no close relationship between the product and the artery. 

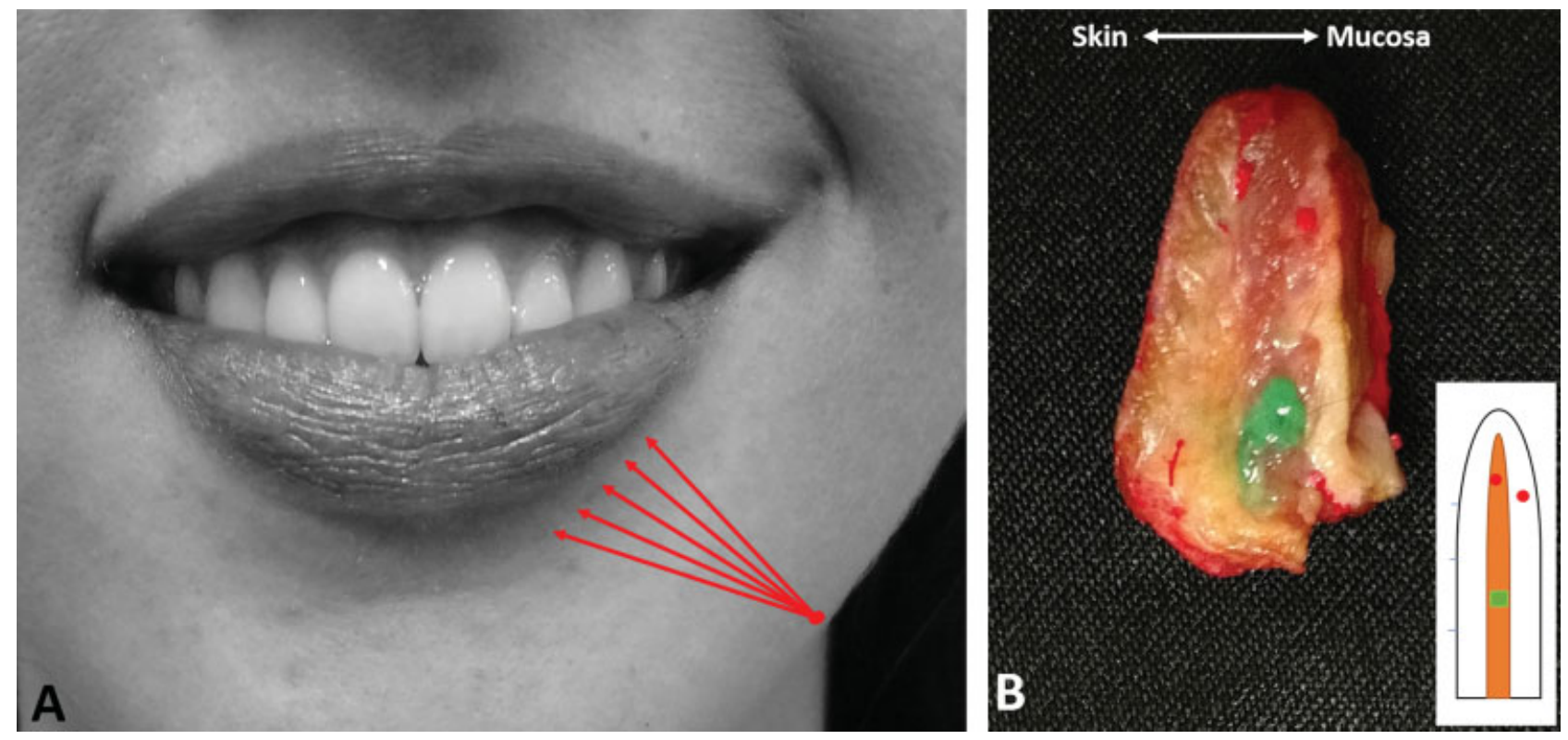

Fig. 12 (A, B) Part A shows the lips of a patient. The colored arrows indicate the point of injection and the direction of the product displacement. The red color represents the treatment with a cannula. Part B shows a cross-section of an injected lip. The green color represents the stained injected product. The red color represents the arteries. The small box in the right lower corner shows a schematic drawing of the lip, the product and the artery, and their position toward each other. Note: In this case, there are two arteries. One is located in the submucosal plane. The second one is located in the intramuscular plane. The product is also located in the intramuscular plane, but there is still no close relationship between the product and the artery.

arteries. The placement of the injected material in the vicinity of the superior/inferior labial arteries indicates a high risk for intra-arterial applications, leading to tissue loss (necrosis) and potential end-arterial embolism (potential blindness). In $60.0 \%$ of the cases, applications using a needle placed the injected product in endangered locations, whereas $57.1 \%$ of the cases using cannulas placed the product in endangered locations (i.e., in the vicinity of the superior/inferior labial arteries).

The results of the anatomical analyses, however, need to be interpreted with caution. First, positioning the product in close proximity to an artery does not necessary mean that the product was placed inside that respective vessel. Placing any product inside the lips requires skills and experience. The location where a product can be injected is limited to the reduced thickness of the lips, which holds especially true for aged lips and those needing treatment. Thus, it can be assumed the any product injected has a certain relationship to the labial vasculature, but the precise plane and the used injector will influence the outcome. However, being in the same plane as the artery (submucosal, intramuscular, or subcutaneous) represents a higher risk to potentially penetrate intra-arterially, whereas having the product separated by the orbicularis oris muscle represents a certain degree of safety.

Second, the vasculature of the lips is highly variable. A recent study has reported that three different positions of the labial arteries are possible: submucosal (i.e., between the oral mucosa and the orbicularis oris muscle in $78.1 \%$ of the cases), intramuscular (i.e., between the superficial and deep layers of the orbicularis oris muscle in $17.5 \%$ of the cases), and subcutaneous (i.e., between the skin and the orbicularis oris muscle in $2.1 \%$ of the cases). The variability in changing the respective position along the labial course was $29 \%$ for the total upper and 32\% for the total lower lip. ${ }^{24}$ The present study confirms the results presented in this report as the labial arteries were identified to run in variable positions independent of the upper/lower lip. On the contrary, the presence of multiple labial arteries also indicates that lips are very forgiving in their nature due to their excellent vascularization. Adverse vascular events might be tolerated and masked well before being clinically apparent. This might be one of the reasons why this few adverse vascular events are being reported in relation to the magnitude of performed labial injections for aesthetic purposes. ${ }^{22,25,26}$ It can be stated that injections deep inside the lips, that is, intramuscular or submucosal, for volumizing procedures (independent if conducted with a needle or a cannula), are associated with a higher risk for intra-arterial positioning of the product.

Third, in the present study the lumen of the arteries was filled with latex, which does not allow a needle or a cannula to penetrate inside the vessel. In a clinical scenario, some of the performed injections in this experimental setting might have positioned the material inside the artery. This is represented in the current analysis by a close relationship between product and artery and could be potentially considered as an indicator for a higher risk for an intra-arterial injection.

The injection techniques presented in this study are based on the experience and personal selection of the authors. The authors have assessed, treated, and evaluated for more than 20 years patients from the Middle East and Central Europe when targeting lip contour, lip volume, and labiomental volume loss. Albeit the focus of a specific treatment might be the lips, which can be sometime expressed very directly by the consulting patient, it has to be well explained that the 
perioral region needs to be addressed first and later the lips themselves. Lip treatment is a multistep approach. Perioral volume loss, perioral wrinkles, and even midfacial or upper facial corrections should be assessed first (if agreed by the patient). Perioral treatment options can influence significantly the positioning of the lips (cranial repositioning after addressing the nasolabial sulcus) and the volume of the lips (increased eversion after treating the deep midfacial fat compartments due to influence of the levator labii superioris alaeque nasi muscle). Age-related changes in the perioral region are caused by a combination of several factors including changes in bony structures (loss of alveolar process), loss of subcutaneous volume (fatty deflation theory), skin laxity (loss of collagen and elastic fibers), and skin sagging (gravity induced). These changes can cause ptosis of the oral commissures, resulting in an inferiorly positioned and deflated labiomental region. The formation of a prominent labiomandibular sulcus (i.e., the marionette lines) can likewise influence the appearance of the lips due to the close relationship to the corners of the mouth.

Middle Eastern females have in general good labial volume, whereas Central European females in the same age group have slightly less volume. Thus, the treatment should focus here rather on contouring than on volumizing. Applying volume in the white (skin covered) and red lips forms a subcutaneous scaffold that stabilizes the lips and provides support for the lower third of the face. Based on the cadaveric part of the present study, it should be noted that $58.3 \%$ of the volumizing procedures were related to a potential intra-arterial injection because the product was injected deep inside the body of the lips. On the contrary, procedures aiming for contouring can be regarded as safer as in this case, the product was positioned in the subcutaneous plane which is separated from the arteries by the orbicularis oris muscle.

\section{Conclusion}

Based on the experience of the authors, the treatment of the lips should be a multistep approach focusing first on the far (upper and middle face) and close (labiomandibular and labiomental) perioral regions. Individuals with an acceptable lip volume should be directed more toward the treatment of the lip contours, whereas individuals with volume deficiency should have first the volume and secondary the contours addressed. The lips are a potential danger zone as $58.3 \%$ of the performed cadaveric injections were in close proximity to the superior/inferior labial artery (and its branches), representing an elevated risk for tissue loss (necrosis) and potential end-arterial embolism (potential blindness).

\section{Funding}

This study received no funding

\section{Conflicts of Interest}

None of the other authors listed have any commercial associations or financial disclosures that might pose or create a conflict of interest with the methods applied or the results presented in this article.

\section{References}

1 Burrows AM. The facial expression musculature in primates and its evolutionary significance. BioEssays 2008;30(03):212-225

2 Kollipara R, Walker B, Sturgeon A. Lip measurements and preferences in Asians and Hispanics: a brief review. J Clin Aesthet Dermatol 2017;10(11):19-21

3 Mannino GN, Lipner SR. Current concepts in lip augmentation. Cutis 2016;98(05):325-329

4 Przylipiak M, Przylipiak J, Terlikowski R, Lubowicka E, Chrostek L, Przylipiak A. Improvements in the perception of facial attractiveness following surgical aesthetic treatment; study based on online before and after photos. J Cosmet Dermatol 2019;18(01): 296-300

5 Przylipiak M, Przylipiak J, Terlikowski R, Lubowicka E, Chrostek L, Przylipiak A. Impact of face proportions on face attractiveness. J Cosmet Dermatol 2018;17(06):954-959

6 Dayan SH, Bacos JT, Gandhi ND, Ho T-VT, Kalbag A. Assessment of the Impact of Perioral Rejuvenation with Hyaluronic Acid Filler on Projected First Impressions and Mood Perceptions. Dermatologic Surg 2019;45(01):99-107

7 Winslow C. Surgical and nonsurgical perioral/lip rejuvenation: beyond volume restoration. Clin Plast Surg 2018;45(04): 601-609

8 Segall L, Ellis DAF. Therapeutic options for lip augmentation. Facial Plast Surg Clin North Am 2007;15(04):485-490, vii

9 Dickinson BP, Roy I, Lesavoy MA. Temporalis fascia for lip augmentation. Ann Plast Surg 2011;66(02):114-117

10 Werschler WP, Fagien S, Thomas J, Paradkar-Mitragotri D, Rotunda A, Beddingfield FC III. Development and validation of a photographic scale for assessment of lip fullness. Aesthet Surg J 2015;35(03):294-307

11 Charles Finn J, Cox SE, Earl ML. Social implications of hyperfunctional facial lines. Dermatol Surg 2003;29(05):450-455

12 Penna V, Stark GB, Eisenhardt SU, Bannasch H, Iblher N. The aging lip: a comparative histological analysis of age-related changes in the upper lip complex. Plast Reconstr Surg 2009;124(02): 624-628

13 Penna V, Stark GB, Iblher N. Aging changes of the male lips-a lesser evil than in females? Ann Plast Surg 2017;78(03): 334-337

14 Luthra A. Shaping lips with fillers. J Cutan Aesthet Surg 2015;8 (03):139-142

15 Byrne PJ, Hilger PA. Lip augmentation. Facial Plast Surg 2004;20 (01):31-38

16 Sarnoff DS, Gotkin RH. Six steps to the "perfect" lip. J Drugs Dermatol 2012;11(09):1081-1088

17 Clymer MA. Evolution in techniques: lip augmentation. Facial Plast Surg 2007;23(01):21-26

18 Maloney BP, Truswell W IV, Waldman SR. Lip augmentation: discussion and debate. Facial Plast Surg Clin North Am 2012;20 (03):327-346

19 San Miguel Moragas J, Reddy RR, Hernández Alfaro F, Mommaerts MY. Systematic review of "filling" procedures for lip augmentation regarding types of material, outcomes and complications. J Craniomaxillofac Surg 2015;43(06):883-906

20 Townshend A. Blindness After Facial Injection. J Clin Aesthet Dermatol 2016;9(12):E5-E7

21 Walker L, King M. This month's guideline: visual loss secondary to cosmetic filler injection. J Clin Aesthet Dermatol 2018;11(05): E53-E55

22 Beleznay K, Carruthers JD, Humphrey S, Jones D. Avoiding and treating blindness from fillers: a review of the world literature. Dermatol Surg 2015;41(10):1097-1117 
23 Loh KT, Chua JJ, Lee HM, et al. Prevention and management of vision loss relating to facial filler injections. Singapore Med J 2016;57(08):438-443

24 Cotofana S, Pretterklieber B, Lucius R, et al. Distribution pattern of the superior and inferior labial arteries: impact for safe upper and lower lip augmentation procedures. Plast Reconstr Surg 2017;139 (05):1075-1082
25 Goodman GJ, Clague MD. A rethink on hyaluronidase injection, intraarterial injection, and blindness: is there another option for treatment of retinal artery embolism caused by intraarterial injection of hyaluronic acid? Dermatol Surg 2016;42(04):547-549

26 Carruthers JD, Fagien S, Rohrich RJ, Weinkle S, Carruthers A. Blindness caused by cosmetic filler injection: a review of cause and therapy. Plast Reconstr Surg 2014;134(06):1197-1201 